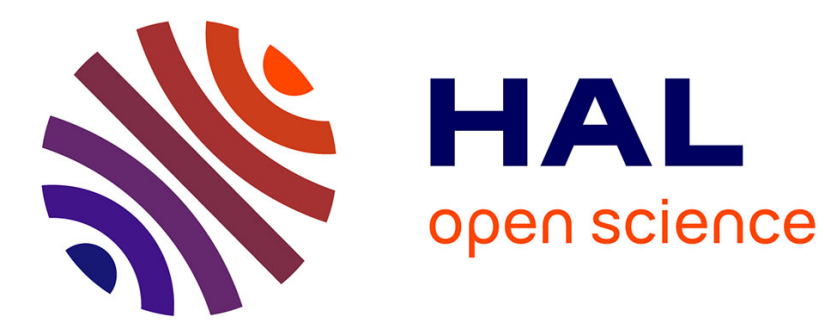

\title{
The IR-RF alpha-efficiency of K-feldspar
}

Sebastian Kreutzer, Loïc Martin, Stéphan Stephan Dubernet, Norbert Mercier

\section{To cite this version:}

Sebastian Kreutzer, Loïc Martin, Stéphan Stephan Dubernet, Norbert Mercier. The IR-RF alpha-efficiency of K-feldspar. Radiation Measurements, 2018, 120, pp.148-156. 10.1016/j.radmeas.2018.04.019 . hal-01846076

\section{HAL Id: hal-01846076 https://hal.science/hal-01846076}

Submitted on 20 Dec 2021

HAL is a multi-disciplinary open access archive for the deposit and dissemination of scientific research documents, whether they are published or not. The documents may come from teaching and research institutions in France or abroad, or from public or private research centers.
L'archive ouverte pluridisciplinaire HAL, est destinée au dépôt et à la diffusion de documents scientifiques de niveau recherche, publiés ou non, émanant des établissements d'enseignement et de recherche français ou étrangers, des laboratoires publics ou privés.

\section{(ㄷ)(1) $\$$}

Distributed under a Creative Commons Attribution - NonCommercial| 4.0 International 
1 Antibacterial, anti-biofilm activity and mechanism of action of Pancreatin doped Zinc oxide nanoparticles against methicillin resistant Staphylococcus aureus.

3 Satarupa Banerjee ${ }^{1}$, KumariVishakha ${ }^{1}$, Shatabdi Das ${ }^{1}$, Debolina Mukherjee, Jyotsna Mondal, Sandhimita Mondal ${ }^{1}$, Arnab Ganguli $^{1^{*}}$

* To whom correspondence should be addressed:

\section{Highlights:}

- Pancreatin coated Zinc oxide nanoparticles (ZnNPs-PK) were prepared and characterized

- ZnNPs-PK shows bactericidal, anti-biofilms anti-motility and anti-virulence activity against methicillin resistant Staphylococcus aureus (MRSA)

- ZnNPs-PK treatment makes MRSA more sensitive to vancomycin.

- ZnNPs-PK targets cell membrane and induced ROS generation as mode of action against MRSA. 


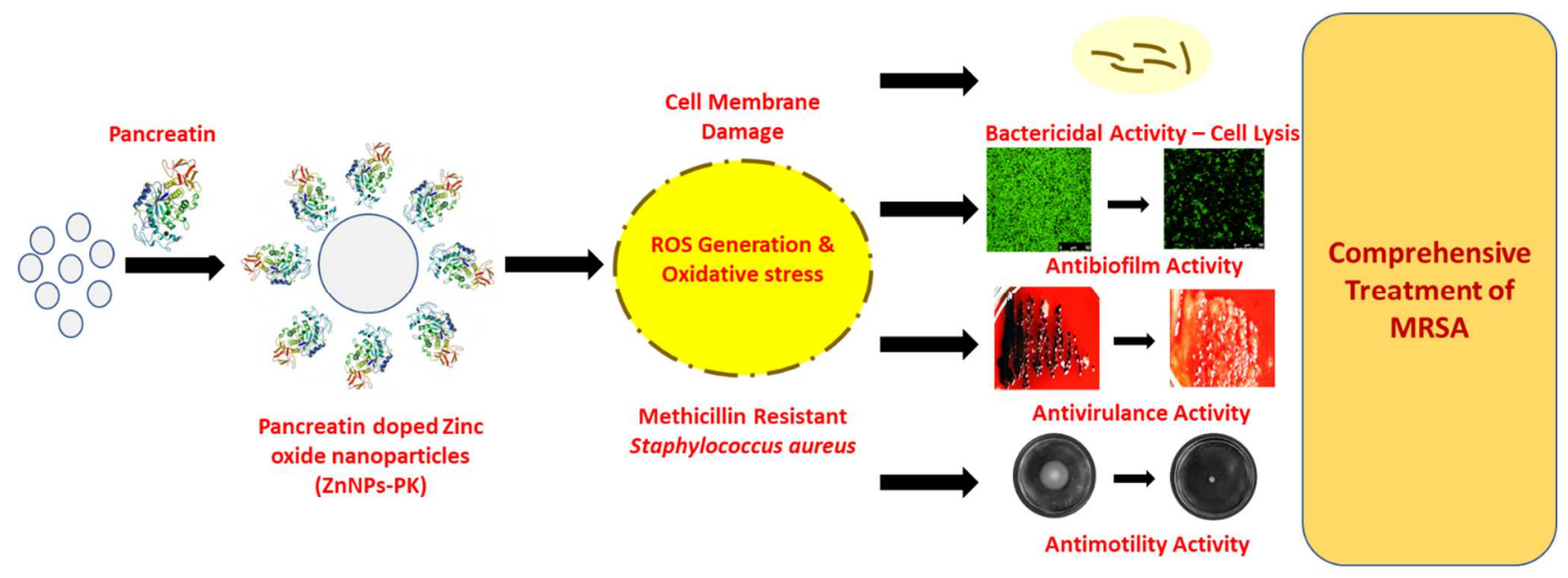

23 


\section{Abstract}

36 Staphylococcus aureus are known to cause diseases from normal skin wound to life

37 intimidating infections. Among the drug resistant strain, management of methicillin resistant 38 Staphylococcus aureus (MRSA) is very difficult by using conventional antibiotic treatment.

39 In present study we show that functionalization of zinc-nanoparticles with pancreatin enzyme

40 have anti-bacterial, anti-biofilms, anti-motility and anti-virulence properties against MRSA.

41 Application of the produced nano-composites as treatment on infected swine dermis

42 predominantly reflects the potential treatment property of it. The vancomycin sensitivity of 43 MRSA was significantly increased on application with ZnNPs-PK. Further study revealed 44 ZnNPs-PK targets bacterial cell membrane and induced oxidative damage as its biocidal 45 mode of action. The produced nanoparticles were found completely non-toxic to human's

46 keratinocytes at its bactericidal concentration. Overall, this study emphasizes the potential 47 mechanisms underlying the selective bactericidal properties of Pancreatin doped zinc oxide nanoparticles against MRSA. This novel nanoparticle strategy may provide the ideal solution

49 for comprehensive management of MRSA and its associated diseases with minimising the 50 use of antibiotics.

51 Keywords: Zinc-oxide-Nanoparticles; pancreatin; MRSA; Selective-bactericidal; Anti52 biofilms; 


\section{Introduction}

59 Staphylococcus aureus is a normal micro flora present in humans and results into an

60 opportunistic pathogen with respect to some immuno-suppresive host circumstances[1]. $S$.

61 aureus infections include extremities from normal skin wound to life intimidating infections

62 like pneumonia, and exotoxins syndromes, endocarditis, and septicaemia[1]. Extended and

63 abandoned usage of bacteriostatic or bactericidal antibiotics has also resulted in the

64 emergence of multidrug resistance strains of S. aureus (MDR)[2]. Amongst the drug resistant

65 strains, methicillin resistant Staphylococcus aureus (MRSA) is an exemplar of third

66 generation antibiotic defiant bacteria. The presence of the mecA gene in the staphylococcal

67 cassette chromosome mec is responsible for the methicillin resistant. MRSA could cause broader infection across communities inside and outside of the hospital[3]. MRSA are found to be more resistant to antimicrobial agent than other nosocomial pathogens[4]. Therefore, it becomes tough to be relieved from the infection associated with MRSAusing conventional antibiotic treatment. This could be a reason why infections associated with MRSA have reached pandemic extent worldwide[5].

73 The existence of various virulence factors such as slime production, exopolysaccharide production, gliding motility, staphyloxanthin pigment production, etc. in $S$. aureus might enhance their probable intimidation particularly that majority of them have enhanced pathogenicity with multi-drug refusal, results it complicated to treat[6]. The most crucial virulence properties of bacteria are the biofilms formation. Biofilms have immense significance for civic wellbeing because of their significant role in spreading of certain contagious diseases[7]. Biofilms associated bacteria are considerably more adaptable to ecological stresses or toxic substances like antibiotics and biocides, than planktonic cells[8].

81 Therefore, for comprehensive treatment of $M R S A$ associated disease, it is important that 82 antibacterial agent should have anti-biofilms and anti virulant properties as well. 
83 Recently, Metal nanoparticles are emerged as a new weapon to combat different bacterial

84 diseases [9]. According to the different metal nanoparticles, Zinc oxide ( $\mathrm{ZnO})$ nanoparticles

85 are reported to low cost, bio-safe and much less toxic for human use [10]. Zinc oxide

86 nanoparticles are reported to have broad spectrum antimicrobial activities even act against

87 different drug resistant strains of pathogenic bacteria including MRSA [11]. Therefore, zinc

88 oxide nanoparticles may consider as an alternative to conventional antibiotics for the

89 treatment of diseases caused by MRSA.

90 On the other hand, enzymes like Pancreatin also play as great anti-microbial agents[12].

91 Pancreatin made up of combination of three enzymes - amylase, protease and lipase. It has

92 been reported earlier that, individually these enzymes eliminate biofilms of MRSA[13]-[15].

93 In addition, this may be a reason for anti-biofilms activity of pancreatin against MRSA.

94 Here in this study, we may hypothesize that $\mathrm{ZnO}$ nanoparticles and pancreatin should be a

95 great combination for comprehensive management of MRSA associated diseases. For this

$96 \mathrm{ZnO}$ nanoparticles were daubed with pancreatin enzymes (ZnNPs-PKs).

97 Our main objective of this study is to produce a pancreatin daubed zinc oxide nano-

98 composites which produce less toxicity but superior anti-bacterial, anti-biofilm and anti-

99 virulent activity against $M R S A$. To achieve that, in this study we first prepared and

100 characterized ZnNPs-PKs and then investigated how ZnNPs-PKs (I) inhibits growth, (II)

101 affects biofilm formation and other virulence factors of MRSA[16]. 


\subsection{Micro-organisms:}

104 Clinically isolated culture of methicillin resistant Staphylococcus aureus collected from 105 Department of Medical Microbiology, Nil Ratan Sircar Medical College \& Hospital, Kolkata,

106 West Bengal, India and maintained in Department of Microbiology, Techno India University,

107 West Bengal was used by growing the bacterial culture on Luria agar, and sub-cultured on

108 Luria Broth prior to each experiment by adjusting turbidity to 0.5 O.D.

\section{2.2. Chemicals and compositions:}

110 Analytical grade zinc nitrate $\mathrm{Zn}\left(\mathrm{NO}_{3}\right)_{2} \cdot 6 \mathrm{H}_{2} \mathrm{O} ;($ Sigma-Aldrich-14436) and potassium

111 hydroxide (KOH) (Merck's global life science, Millipore Corporation, USA). Hydrazine 112 hydrate $\left(\mathrm{N}_{2} \mathrm{H}_{4} ; 98.0 \%\right)$ of Sigma-Aldrich (St Louis, MO, USA). Crystal violet solution (99\%)

113 of (Merck Merck's global life science, Millipore Corporation, USA) which was used to stain 114 biofilms. 96-wells plates and 12-wells plates (Tarsons Products (P) Ltd.) were used of 115 biofilms inhibition assay.

\section{2.3. Synthesis and characterization of nano-composites:}

117 Synthesis of Zinc oxide nanoparticles were prepared by following established protocol [17].

118 At first, Zinc oxide nanoparticles (ZnNPs) were prepared by precipitation method. Potassium 119 hydroxide solutions $(\mathrm{KOH})(0.4 \mathrm{M})$ were slowly added with Zinc nitrate $(0.2 \mathrm{M})$ in deionised 120 water at room temperature. The solution was mixed by vigorously stirring until it formed 121 turbid white suspension. The produced white milky suspension was centrifuged at $5000 \mathrm{rpm}$ 122 for 20 minutes and washed thrice with deionised water. Pellet was further washed with 123 ethanol. The resultant product was then calcined in air atmosphere at $500^{\circ} \mathrm{C}$ for $3 \mathrm{hr}$. For the 124 preparation of pancreatin daubed zinc oxide nanoparticles (ZnNPs-PK), $2 \mathrm{mg} / \mathrm{ml}$ pancreatin 
125 enzyme solution was added to the $10 \mathrm{mg} / \mathrm{ml}$ zinc oxide nanoparticles solution. This was then

126 stirred vigorously under room temperature for $2 \mathrm{hrs}$. After that suspension was centrifuged at

$1275000 \mathrm{rpm}$ for 20 minutes and washed thrice with deionised water. The prepared nano-

128 composites were the characterized by following way.

129 To observe the optical property of prepared nanocomposites, samples were analysed for UV-

130 vis spectroscopic studies (UV-visible Spectrophotometer 2206, SYSTRONICS) at room

131 temperature operated at a resolution of $1 \mathrm{~nm}$ between 200 to800 $\mathrm{nm}$ ranges Dynamic Light

132 Scattering (DLS) analysis was done with a Zeta sizer Nano ZS (Malvern Instruments)

133 according to standard method with some modifications. The amount of enzyme adsorbed on

134 the ZnNPs-PK surface was evaluated by Bradford assay using UV-VIS spectrophotometer.

\section{2.4. Antibacterial activity of ZnNPs-PK}

136 2.4.1 Determination of minimal inhibitory concentration (MIC) and Minimum

\section{7 bactericidal concentration (MBC):}

138 Determination of minimal inhibitory concentrations (MICs) ofZnNPs and ZnNPs-PK for

139 MRSA was determined according to standard protocol lThe bacterial suspension were treated

140 with or without different concentration of ZnNPs-PKs and were incubated at $37^{\circ} \mathrm{C}$ overnight

141 under shaking condition. After incubation, the MIC values were obtained by checking the

142 turbidity of the bacterial growth. The MIC value corresponded to the concentration that

143 inhibited $99 \%$ of bacterial growth[18].

144 Minimum bactericidal concentration (MBC) was estimated by spreading plating from MIC 145 assay and incubated overnight. The lowest concentration of the nanoparticles which 146 completely killed the tested bacteria was observed and tabulated as MBC value[18].

\section{$147 \quad$ 2.4.2 Death kinetics:}


148 To determine the death rate of MRSAin presence of ZnNPs-PK, an overnight culture was

149 diluted 100 times in fresh LB and allowed to cultivate at $37^{\circ} \mathrm{C}$ under shaking condition upto

$150 \log$ phase $\left(\sim 5 \times 10^{7}\right.$ cells/ml). ZnNPs-PK was supplemented at MBC and the cells were

151 incubated overnight at $37^{\circ} \mathrm{C}$ under shaking condition. At different time interval of 60 mins,

152 cell aliquots were withdrawn, serially diluted and spread on agar plates to evaluate quantity

153 of viable cells[19][20]. According to the slope of the plot ( $\log \mathrm{N}_{\mathrm{t}} / \mathrm{N}_{0}$ against time associated

154 with incubation), the death rate of ZnNPs-PK treated cells was evaluated[18].

\section{$155 \quad 2.5$ Anti-biofilms activity of ZnNPs-PK}

\section{$156 \quad 2.5 .1$ Initial biofilms inhibition:}

157 In brief, overnight cultures of MRSA were inoculated into wells of a polystyrene 24-well cell

158 culture plate with LB containing various concentrations of ZnNPs-PK and then incubated at

$15937^{\circ} \mathrm{C}$ for $24 \mathrm{~h}$ without agitation. After incubation, LB was removed, and the wells were

160 thoroughly washed with sterile phosphate-buffered saline (PBS) to remove planktonic and 161 non-adherent cells.

162 For determination of biofilm biomass sessile cells were stained with $0.05 \%$ crystal violet, the

163 excess of which was then rinsed off using distilled water. After dissolution with $95 \%$ ethanol,

164 the biofilm biomass was determined by reading OD595. The percentage biomass formation

165 was determined using the following equation.

166 Percentage Biofilm Formation $=[\{$ Test Sample OD595 nm/Control sample OD595 nm $\}$

$167 \times 100]$.

168 To investigate cell viability, adherent bacteria in each well were resuspended by vigorous

169 pipetting and vortexing followed by sonication and then were serially diluted $10^{6}$ - through

$17010^{8}$-fold and then plated onto LA plates. The agar plates were incubated at $37^{\circ} \mathrm{C}$ for $24 \mathrm{~h}$

171 before bacterial colonies were counted. 


\subsubsection{Mature biofilms degradation:}

173 Biofilms were cultivated in LB broth in 24 -well polystyrene plates at $37^{\circ} \mathrm{C}$ without shaking.

174 After 24hrsincubation, the broth were removed and the biofilms were rinsed with PBS and 175 then supplemented with fresh LB broth and ZnNPs-PK with different concentrations[20].

176 After another 24-h cultivation, the formed biofilms were washed with PBS and stained using

177 crystal violet, solubilized with ethanol, and eventually quantified at $595 \mathrm{~nm}$ using a 178 microplate reader.

179 For cell viability, sessile cells were washed with PBS, then resuspended by vigorous pipetting 180 and vortexing and then sonicated for $30 \mathrm{~s}$. The number of CFU/biofilm was quantified by LB 181 agar plating.

182

183 2.5.3 Microscopic observation of biofilm:

\subsubsection{Light Microscopy}

185 For light microscopy, the biofilm assay was performed with small glass slides $(1 \times 1 \mathrm{~cm})$

186 placed in the wells of the 24-well polystyrene plate. ZnNPs-PK was added at its MIC to the 187 preformed biofilms incubated at $37^{\circ} \mathrm{C}$ for $24 \mathrm{~h}$. After incubation, planktonic cells were 188 removed, and the biofilm formed on the glass slides was stained using crystal violet dye for 5 189 min. It was then gently washed with PBS and allowed to dry for $5 \mathrm{~min}$. Then, the slides were 190 viewed under a light microscope at 40x magnification and images were taken using digital 191 camera[21].

\section{$192 \quad$ 2.5.3.2 Confocal Microscopy}

193 The surface topology of MRSA biofilm architecture were visualized under Confocal Laser

194 Scanning Microscope (CLSM) by forming biofilm on glass slide placed on 24-well 
195 polystyrene microtiter plate[20]. To determine the effect of ZnNPs-PK to disrupt the mature

196 biofilms, MIC of ZnNPs-PK was added to the mature biofilms and incubated overnight at

$19737^{\circ} \mathrm{C}$, then the glass slides were taken and washed with PBS followed by staining with

198 acridine orange and observed using CLSM (Carl Zeiss LSM700, Jena, Germany)[21].

\section{2.6. Anti-motility assay:}

$200 \quad$ 2.6.1 Sliding motility:

201 The spreading ability of MRSA on soft LB agar was assessed in the presence and absence of

202 ZnNPs-PK. LB soft agar was prepared with $24 \mathrm{~g} / 1$ lit of agar and poured into the petri dish 203 plate. Overnight, treated (MIC concentration) and un-treated cultures of MRSA were spotted

204 in the middle of the plate and air dry for 20 mins at room temperature and incubated for 48

205 hours at $37^{\circ} \mathrm{C}$. Sliding motility of selected strains was estimated through measuring the 206 expansion of colony growth from the inoculation point[21].

\section{2.6.2 Colony spreading assay:}

208 To determine the anti-colony-spreading activity of ZnNPs-PK $5 \mathrm{ml}$ of LB agar (0.4\%)

209 medium with MIC of ZnNPs-PK and without ZnNPs-PK was prepared and then poured over

210 the LB agar plates (2\%). After solidification, MRSA were spotted on the centre of the plate

211 and incubated for $24 \mathrm{hrs}$ at $37{ }^{\circ} \mathrm{C}$. Further, the diameter of the colony was measured to

212 determine the effect of ZnNPs-PK on spreading[21].

2132.7 Anti-virulence study:

\section{2.7.1 Slime production:}

215 The ability ZnNPs-PK to reduce slime synthesis of MRSA was determined by Congo red agar 216 (CRA) analysis. ZnNPs-PK at its MIC was streaked to CRA and was incubated at $37^{\circ} \mathrm{C}$ for 
21724 hours. After incubation the changes from black colonies to Bordeaux red colonies was

218 noted. The process was practiced in triplicates[22].

219 2.7.2 Exopolysaccharide production:

220 To determine EPS production, MRSA culture with and without MIC of ZnNPs-PK was grown 221 on LB media in 24 well plate for $24 \mathrm{~h}$ at $28 \pm 2{ }^{\circ} \mathrm{C}$.After incubation, the non-adherent cells

222 were aspirated and removed and $0.5 \% \mathrm{NaCl}$ was added. These cells suspended in $0.5 \% \mathrm{NaCl}$ were transferred to fresh sterile test tubes and added with equal volume of 5\% phenol. To that solution, 5 volume of concentrated sulfuric acid containing $0.2 \%$ hydrazine sulphate was added and incubated in dark for $1 \mathrm{~h}$ and the absorbance was measured at 490nm[23].

\subsubsection{Staphyloxanthin production assay:}

227 The ability of ZnNPs-PK to reduce the staphyloxanthin pigment secretion was investigated by carotenoid extraction. MRSA at its mid log phase were sub-cultured by diluting it to $1: 10$ (V/V) and ended up to an ultimate volume of $5 \mathrm{ml}$ in LB broth. To the culture, ZnNPs-PK at MIC was added and incubated at $37{ }^{\circ} \mathrm{C}$ for 48 hours under shaking conditions. After incubation the cultures were centrifuged at $10,000 \mathrm{rpm}$ for $5 \mathrm{mins}$ at $4^{\circ} \mathrm{C}$. Culture devoid of ZnNPs-PK was considered as control. The change in color of the culture from golden yellow was measured at an absorbance of $462 \mathrm{~nm}$ using UV-VIS spectrophotometer[24].

\subsubsection{Bacterial Hemolysis activity:}

Blood samples of $5 \mathrm{~mL}$ mixed with acid citrate dextrose to avoid clotting. These samples were

236 first centrifuged, removed the supernatant and then diluted with PBS at 1:10 ratio[25].

237 Diluted blood samples were inoculated with un-treated and treated bacterial cultures were 238 added to it[20]. These combinations were incubated overnight at $37^{\circ} \mathrm{C}$. Prior to incubation, 239 the tubes were centrifuged at 3,000 rpm for $20 \mathrm{~min}$. Absorbance of supernatants was 
240 calculated at $540 \mathrm{~nm}$ and the rate of haemolysis was intended in percentage for the triplicate 241 data[22].

\section{2.7.5 Plasma clamping assay:}

243 Overnight cultures of MRSA were re-suspended in PBS. Human blood plasma was removed

244 from RBC by centrifugation at $10,000 \mathrm{rpm}$ for 5 minutes. $20 \mu \mathrm{L}$ of plasma was given on the 245 surface of glass slides as small circular drops. In addition, un-treated and treated MRSA 246 suspension was added to each of the slides having the plasma samples. Blood plasma with

247 un-treated MRSA served as positive control, and plasma with application of distilled water is 248 served as a negative control. The suspensions were blended uniformly macroscopic 249 agglutination of bacterial cells was observed in each trice times[26].

\section{$250 \quad 2.8$ Ex-vivo porcine skin model}

251 Porcine skin majorly includes Swine dermis. Porcine samples were collected from excess 252 trash of local meat shop. The sample was cut in appropriate dimensions $(1 \times 1 \mathrm{~cm})$ and was 253 surface sterilised with $1 \%$ isopropanol for 2 hours. The sample skins were place aseptically in 254 sterile stainless-steel clamps having two to three holes for inoculations. Treated and untreated 255 bacterial samples with or without ZnNP-PK were inoculated in the holes of the clamps at 256 MIC and sub-MIC concentrations. Samples treated with sterile media broth were served as 257 negative controls and samples applied with untreated MRSA were served as positive or 258 infective controls. The setups were incubated for $48-72$ hours at $37^{\circ} \mathrm{C}$. Each setup was 259 experimented in triplicate.

\subsection{Mechanisms of action:}

\subsubsection{ROS generation:}


262 ROS generation in nanoparticles treated cells were investigated using flow cytometric 263 technique. The $\mathrm{DCFH}_{2}$-DA invade the cell of MRSA and combines with ROS to build the

264 highly fluorescent composite 2,7-dichlorofluorescein. After treatment with nanoparticles, 265 MRSA were cultured and flashed thoroughly with PBS. The cell pellet was taken and a 266 homogenous suspension up to $1 \mathrm{~mL}$ was prepared by PBS buffer[27]. Subsequently, the cells

267 were incubated with $1.5 \mathrm{~mL}$ of $100 \mu \mathrm{M} \mathrm{DCFH}_{2}-\mathrm{DA}$ for $30 \mathrm{~min}$ at a temperature of $37^{\circ} \mathrm{C}$. The 268 ROS production was evaluated by flow cytometry (Model: FACSVerse flow cytometer, 269 Becton Dickinson). Data were evaluated by FCSExpress Software.

\subsubsection{Membrane potential change:}

271 Membrane depolarisation was measured by Rhodamine-123 (Rh123) dye. After treatment

272 with nanoparticles, MRSA were cultured and flashed thoroughly with PBS. The cell pellet 273 was taken and a homogenous suspension up to $1 \mathrm{~mL}$ was prepared by PBS buffer. Rh123 274 reagent was added and incubated in the dark for 10 minutes and then analysed by flow 275 cytometry (Model: FACSVerse flow cytometer, Becton Dickinson). Data analysed by 276 FCSExpress Software.

\section{2.9.3 Membrane Damage:}

278 Propidium Iodide (PI) can enter the bacterial cell membrane only when it has been 279 permeabilized through an agent, and bind with DNA and gives fluorescence. The 280 fluorescence emission can be detected by flow cytometer. After treatment the cells were 281 washed in PBS buffer and incubated with PI $(1.3 \mu \mathrm{g} / \mathrm{ml})$ at $37^{\circ} \mathrm{C}$ for $20 \mathrm{~min}$ in dark. The PI

282 fluorescence was measured in the flow cytometer (Becton Dickinson (BD) FACSVerse). 283 Data analysed by FCSExpress Software. 
285 Combination activity of ZnNPs-PK combined with commercial drugs of vancomycin was 286 evaluated against MRSA. For this we first determined the MIC of vancomycin against

287 MRSA. Then MRSA were treated with $1 / 4$ and $1 / 2$ MIC concentration of ZnNPs-PK and 288 vancomycin individually and in combination. The inhibitory activity was determined by 289 checking the turbidity of the bacterial growth. This was measured by spectrophotometrically 290 at a wavelength of $600 \mathrm{~nm}$.

\subsection{Cytotoxicity study on Keratinocytes}

292 HaCaT cells were seeded in 96-well plates at a density of $10^{4}$ cells per well in $0.2 \mathrm{ml}$ of 293 DMEM: Ham's F12 (1:1 v/v) with10\% FBS and 1\% antibiotics and was cultured at 5\% $\mathrm{CO}_{2}$ 294 and $37{ }^{\circ} \mathrm{C}$ for $24 \mathrm{~h}$ [30]. Growth medium inthe wells was replaced after $24 \mathrm{~h}$ with medium 295 containing different concentration of ZnNPs-PK and incubated for $24 \mathrm{~h}$. The medium was 296 removed thereafter and replaced with100 $\mu$ l of medium containing 3-(4, 5-dimethyl-thiazol-2297 yl)-2,5-diphenyltetrazolium bromide (MTT) and incubated for4 h. The unreduced MTT was 298 taken out, and $200 \mu \mathrm{l}$ of DMSO was added to each well to dissolve the MTT formazan 299 crystals. The content was mixed properly, and absorbance was measured at $595 \mathrm{~nm}$ in a 300 microplate reader.

301 3. Results:

\section{3.1. Characterisation of nanoparticles:}

303 The absorption spectra of the ZnNPs (Fig.1A) had a broader band with a maximum at 342nm.

304 After doping with pancreatin we also spectrophotometrically characterized the nanoparticles.

305 The absorption spectrum of ZnNPs-PK showed an increased absorption at 280nm indicating 306 the binding of enzyme to the ZnNPs nanoparticles. 
307 Average particle size, distribution and polydispersity index (PDI) of synthesized Zn-NPs and 308 ZnNPs-PK in solutions were evaluated by DLS technique. The DLS pattern revealed that

309 ZnNPs had a $\mathrm{Z}$ average diameter of $5 \mathrm{~nm}$. Which was significantly increased to $45 \mathrm{~nm}$

310 (Fig.1B). Hence it may hypothesize that binding of pancreatin to ZnNPs increased the

311 particles size of the nanoparticles. The PDI value of the synthesized ZnNPs was 0.641

312 indicating nearly monodisperse distribution. Little change in the PDI value was observed

313 after the binding with pancreatin, indicating that the size distribution was not affected by the

314 ZnNPs-PK interaction.

315 The percent of protein adsorbed on the functionalized nanoparticles surface was $0.47 \pm 0.4 \%$

$316(\mathrm{~W} / \mathrm{W})$ as determined by Bradford assay. That indicates binding of few micrograms of

317 proteins per milligram of nanoparticles.

$318 \quad 3.2$ Antibacterial activity of ZnNPs-PK:

319 3.2.1 Determination of minimum inhibitory concentration of ZnNPs and ZnNPs-PK 320 against MRSA

321 At first, we determined comparative antibacterial activity of ZnNPs and ZnNPs-PK against

322 the MRSA (Fig.2A). For this, we did broth dilution method to determine the minimum

323 inhibitory concentration of ZnNPs and ZnNPs-PK against the MRSA. Different

324 concentrations of the nano-composites were used to treat MRSA for the estimation of the MIC

325 value. After incubation, spectrophotometric analysis revealed that the MIC90 for ZnNPs and

326 ZnNPs-PK was 75 and $156 \mu \mathrm{g} / \mathrm{mL}$ respectively. This indicates ZnNPs-PK nanoparticles

327 exhibit greater antimicrobial potential than ZnNPs. Thus ZnNPs-PK nano-composites were

328 used to study further antibacterial, anti-biofilms and anti virulence activities against MRSA. 
329 To understand the mode of antimicrobial activity, MBC concentration of ZnNPs-PK against

330 MRSA was determined. The MBC concentration was found to be $120 \mu \mathrm{g} / \mathrm{mL}$. After that, as

331 previous reports suggests[31], we determined the tolerance value to understand the nature of 332 antimicrobial activity of the nano-composites. The tolerance value was 1.6 indicated 333 bactericidal activities of the ZnNPs- PK against MRSA.

\section{3.2.2 Death kinetics:}

335 As we found prepared nanoparticles are bactericidal in nature, hence definitely these 336 nanocomposites have an effect on the growth of bacteria and that can be evaluated by 337 investigating the death kinetics of MRSA in presence and absence of ZnNPs-PK. For this we 338 treated MRSA with MBC concentration of nanoparticles and subsequently the viable cell 339 counts were measured at different times of exposure up to 6 hours, because significant 340 amount cell killing occur within this period. The rate of death percentage of MRSA after the 341 treatment was calculated from slopes of straight-line curve (Fig.2B) to be $0.87 \mathrm{~h}^{-1}$. In the 342 untreated MRSA the growth rate was $0.72 \mathrm{~h}^{-1}$. This result strengthened the bactericidal nature 343 of our nano-composites.

\section{3.3. Anti-biofilms activity of ZnNPs-PK against MRSA}

\section{3.3.1 Initial biofilms inhibition:}

346 At the initial stage of disease progression, planktonic cells are always trying to attach to the 347 substratum or the surface for colonization. That leads to the formation of biofilm. Therefore, 348 inhibition of this initial attachment may be a key factor for finding promising antibiofilm 349 agents. The effects of ZnNPs-PK on inhibition of initial biofilms formation by MRSA was 350 determined by enumerate viable sessile cells and crystal violet assay. $1 / 2$ MIC and MIC 351 concentration of ZnNPs-PK treatment reduced the viable sessile cells by 4 and $7 \log$ 352 (CFU/well) respectively as compared with control (Fig.3A). Similarly, the biofilms biomass 
was also decreased to 50 and $30 \%$ after treatment with MIC and $1 / 2$ MIC concentration of

ZnNPs-PK. (Fig.3A). We also investigated effect of only ZnNPs on initial biofilms formation of MRSA. We found at $75 \mu \mathrm{g} / \mathrm{ml}$ of ZnNPs treatment could not showed any significant antagonistic effect on survivability of sessile cells of MRSA.

\subsubsection{Preformed biofilms degradation:}

As we found an encouraging result about initial biofilm formation inhibition by ZnNPs-PK, we extended our study to understand the disruptive potential of our prepared nanoparticles on preformed biofilms. Prior treatment, we first incubated MRSA for $48 \mathrm{~h}$ to allow the biofilms formation. The $1 / 2 \mathrm{MIC}$ and MIC concentrations of ZnNPs-PK treatment resulted in a remarkable reduction of the preformed biofilms in MRSA (Fig.3A). Similarly, the sessile cells of preformed biofilms were eradicated by ZnNPs-PK in a concentration-dependent manner (Fig.3B).

\subsubsection{Microscopic observation of biofilms inhibition by ZnNPs-PK:}

366 Both the light and confocal microscopic study confirmed disruption of MRSA biofilms by

367 ZnNPs-PK. For light microscopic study, application of crystal violet on the mature biofilms

368 by MRSA, untreated and treated with ZnNPs-PK at its MIC concentrations. Biofilms 369 disruption was clearly observed as the untreated samples retained more stains than the treated samples (Fig.3B). The consequence of ZnNPs-PK to disturb the mature biofilms of MRSA was also examined with CLSM by using acridine orange staining. It is apparent that there is a

372 decrease of green fluorescence in treated samples when compared to that of control indicating

373 disruption of biofilms. All these data suggest ZnNPs-PK effectively eradicate the bioflim formation of MRSA. 


\subsubsection{Sliding assay:}

377 Gram-positive MRSA is anon flagellated bacteria, hence they can spread by means of sliding 378 motility and that aided to increased biofilm formation on the surface[32]. Thus, to understand 379 the effect of ZnNPs-PK on sliding motility of MRSA was determined by inoculating a culture 380 of MRSA in presence and absence ofZnNPs-PK on the centre of semisolid growth media and 381 incubated for $48 \mathrm{hrs}$ at $37^{\circ} \mathrm{C}$. In control, we found the sliding motility was well established, 382 wherein the plates treated with nanoparticles significantly reduced the sliding motility of 383 MRSA (Fig.4A).

\section{3.4.2 Colony spreading assay:}

385 In colony spreading assay, we found untreated control MRSA appeared as rapid and 386 expanded colony spreading on the agar surface, whereas ZnNPS-PK treated MRSA showed a 387 notable reduction in spreading as compared to control (Fig.4A).

\subsection{Anti-virulence activity of ZnNPs-PK against $M R S A$}

\subsubsection{Exopolysaccharide and Slime production:}

390 The Slime and exopolysaccharide production by bacteria were considered as key virulent

391 factors for attachment, colonization and biofilm formation within host[33]. Therefore, 392 reduction of both slime and exopolysaccharide production is an important criterion for an anti-biofilm agent. In this study, we evaluated the capability of ZnNPs-PK to reduce the slime

394 and exopolysaccharide production of MRSA was analysed by Congo red agar (CRA) test and

395 EPS quantification analysis respectively. In CRA test ZnNPs-PK at its MIC concentration

396 reduced the slime production which was determined by color alteration from black to 397 bordeaux red (Fig.5A). On the other hand, EPS production was spectrophotometrically 
quantified in treated and untreated samples. We found EPS production was significantly reduces in MIC treated MRSA as compared to the control (Fig.6A).

\subsubsection{Staphyloxanthin production assay:}

401 Staphyloxanthin protects MRSA from oxidative stress as well as from the host immune 402 system[34]. Therefore, reduction of staphyloxanthin should be an advantageous for an 403 antimicrobial agent. We found in our study, staphyloxanthin production of MRSA was 404 significantly reduced at MIC treatment of ZnNPs-PK as in Fig.6A. Therefore, it can be 405 hypothesized that ZnNPs-PK makes susceptible MRSA towards oxidative stress.

\section{$406 \quad$ 3.5.3 Bacterial Hemolysis activity:}

407 S. aureus produces a series of haemolysins which lyses erythrocytes and various leukocytes 408 such as neutrophils, monocytes, granulocytes and macrophages[35]. The effectiveness of 409 ZnNPs-PK to reduce the haemolytic virulence property of MRSA strains, erythrocytes was 410 exposed to MRSA with or without treated with ZnNPs-PK. We observed that ZnNPs-PK 411 treated MRSA showed anti-hemolytic activity. Up to $80 \%$ inhibition in haemolytic activity 412 was detected upon treatment with ZnNPs-PK (Fig.6A).

\section{3.5.4 Plasma clamping assay:}

414 Clumping factors are key virulence of MRSA for their pathogenicity and survival against host

415 immune system ${ }^{\circledR}$. In this study, to understand the ability of ZnNPs-PK to inhibit clumping 416 factors of MRSA, we evaluated bacterial cell clumping activity using the slide coagulation test 417 with blood plasma.In the absence of nanoparticles, MRSAwas found to readily agglutinate in 418 blood plasma, whereas in ZnNPs-PK treated MRSA, less clumping activity was observed 419 (Fig.5B). 

against MRSA

Porcine skin surface was inoculated with MRSA with or without treatment at MIC of ZnNPsPK. The skin surface was the cultured $24 \mathrm{~h}$ for biofilms formation and physical appearance of infections (Fig.8A). The established bioburden was characterized by plating to determine $\mathrm{CFU} / \mathrm{ml}$. After $24 \mathrm{~h}$, we noted $9.45 \times 10^{4} \mathrm{CFU} / \mathrm{cm} 2$ in untreated skin surface. However, in MIC of ZnNPs-PK treatment significantly reduced the bioburden of $M R S A$ to $3 \times 10^{4} \mathrm{CFU} / \mathrm{cm}^{2}$.

\subsection{Mechanisms of action of ZnNPs-PK against MRSA}

\subsubsection{ZnNPs-PK induced the oxidative stress in $M R S A$}

Oxidative stress levels in MRSA after treatment with ZnNPs-PK was determined by using the 2',7'-dichlorofluorescin diacetate (DCFDA) assay. We noted that by flow cytometric assay mean fluorescence intensity of green fluorescence in MRSA treated with MIC of ZNNPs-PK was significantly increased as compared with the untreated cells. This indicates ZNNPs-PK induced oxidative stress by means of ROS generation in MRSA and this may be a possible mechanism responsible for the antibacterial effects of nanoparticles (Fig.6B).

\subsubsection{Membrane potential change:}

436 Change in membrane potential after treatment with antimicrobial was considered as another 437 antimicrobial mechanism. Membrane potential has a key role in bacterial physiology[36]. 438 Therefore change in membrane potential is an early onset of injury in bacteria. In our study it 439 was evaluated by flow cytometric assay using rhodamine 123. After exposure to ZnNPs-PK 440 at MIC a considerable decrease of MFI values was observed respectively as compared with 441 control (Fig.7A). 
443 Next to understand the membrane damage; we used propidium iodide-based flow cytometric 444 method. In untreated MRSA, only a small proportion of cells $5 \%$ were stained with PI

445 indicating lesser membrane damage. However, after treatment with MIC of ZnNPs-PK, the 446 bacterial cells with damaged membrane stained by PI were increased to $77 \%$ (Fig.7B).

447 Therefore, it can be hypothesized that ZnNPs-PK targets bacterial cell membrane for its 448 antimicrobial activity.

\subsection{Combination study of ZnNPs-PK with vancomycin against MRSA}

450

\section{Discussions:}

In our study we found the MIC value of vancomycin against MRSA was $1 \mu \mathrm{g} / \mathrm{ml}$. It has been reported that vancomycin MIC against MRSA in the range of 0.5 to $2 \mu \mathrm{g} / \mathrm{ml}$ would have significant clinical implications ${ }^{\circledR}$. Therefore, we performed a combination study to understand the effect of ZnNPs-PK on the vancomycin sensitivity to MRSA. We found $1 / 4$ MIC concentrations of both antibacterial in combination completely inhibit the growth of MRSA as compared with individual treatment (Fig.8B). Therefore, this signifies that ZnNPsPK may intensify the sensitivity of vancomycin to MRSA.

\subsection{Cytotoxicity of ZnNPs-PK on HaCaT (Keratinocytes) cell line}

Cytotoxicity of ZnNPs-PK on HaCaT (Keratinocytes) cell line was determined by MTT assay. The IC90 (concentration at which $90 \%$ decrease in cell viability was observed) value was found to be $400 \mu \mathrm{g} / \mathrm{ml}$. The cytotoxic concentration of nanoparticles was found to be 5fold higher than that of MIC for bacteria (Fig.7C). Selectivity of ZnNPs-PK was calculated by taking the ratio of $\mathrm{IC} 90_{\mathrm{HaCaT}} / \mathrm{MIC}_{\mathrm{MRSA}}$ and it was found to be 5.33 [37].

\section{Organisms like MRSA are primarily known for its skin infections. It is a foremost reason of} bacteraemia and infective endocarditic problems additionally osteo-articular, skin and 
466 flexible tissue damage[38]. As MRSA is considered as a well-known MDR strain, it is 467 tremendously difficult to be treated to normal ranges of antibiotics. Moreover, next 468 generations of antibiotics also impart toxic side effects on human body[39]. The treatment is 469 further complicated as MRSA are known as strong biofilms producer[40]. The biofilms 470 formations not only increase the resistant against the antibiotic but also prevent antagonistic 471 activities of immune cells of the host[41]. Under these circumstances, it has become very 472 important to produce an alternative way which has efficient antimicrobial as well as 473 antibiofilm effect on the respective strain though having low toxicity. In this study, initially 474 we have been able to produce zinc oxide nanoparticles (ZnNPs). ZnNPs are considered as 475 less toxic, biosafe among the other metal nanoparticles[10]. Further, these nanoparticles have 476 been doped with antibacterial enzyme pancreatin. The initial process of this study includes 477 the characterization of the formed nanoparticles which involves analytical tests like DLS, 478 UV-visible spectroscopy. Both DLS and UV spectra analysis assure the presence of doped 479 pancreatin on the surface of zinc-oxide nanoparticles. The lower MIC value of ZnNPs-PK 480 than ZnNPs suggest pancreatin doping increased the antimicrobial potential of nanoparticles. 481 Tolerance value indicated biocidal activity of ZnNPs-PK, which is further confirmed by 482 death kinetics assay. Inhibition of biofilm formation of pathogenic organisms is also an 483 important criterion for a comprehensive antimicrobial agent [42]. ZnNPs-PK was found to impaired initial and mature biofilms formation of MRSA. It can be hypothesized that, 485 amylase lipase and protease activity of pancreatin degrade the carbohydrate and protein rich 486 biofilms produced by MRSA. After degradation of the biofilms it become easier for the 487 ZnNPs to invade and shows antagonistic activity on the sessile bacterial cell. Motility is an 488 important criterion of bacteria for its initial attachment with substratum and following biofilm 489 formation to disease progression[32]. Therefore, antimotility activity of antibacterial agent 490 should provide an additional advantage for disease management. In our study, ZnNPs-PK 
491 inhibit both sliding motility and colony spreading activity of staphylococcus and may 492 consequently inhibit the biofilm formation as reported previously. Like motility, EPS 493 production also plays an important role for bacterial adhesion, adaptation and biofilm 494 formation [33]. EPS production significantly reduced in ZnNPs-PK treated MRSA, which 495 might be the reason for the reduction of biofilm of MRSA.MRSA produce variety of 496 virulence factors for the disease progression [43]. Therefore, inhibition of one or two 497 virulence factors is not enough for a comprehensive treatment. ZnNPs-PK was also found to 498 diminish staphyloxanthin secretion and eventually increase susceptibility of MRSA to 499 oxidative stress and host immune system. Not only that, other two virulence factors those are 500 important for survival within host, such as the haemolytic and plasma clumping activity were 501 significantly reduced in ZnNPs-PK treated MRSA give an idea of anti-infective nature of the nanoparticles. This anti-infective nature of nanoparticles was further assessed by porcine skin model. Where we found bioburden was significantly reduced in ZnNPs-PK treated MRSA 504 infected skin model as compared with control. All these data suggest ZnNPs-PK is an 505 effective antibacterial, anti-biofilms, anti-virulence and anti-infective agent against MRSA.

506 Furthermore, it is important to understand the mechanism associated with antibacterial 507 activities of ZnNPs-PK on MRSA. It has been previously reported that ZnNPs induced ROS 508 generation and targets bacterial membrane [44]. In our study we also found ZnNPs-PK 509 treatment induced significant reduction of membrane potential indicating it may target 510 bacterial cell membrane. Bacterial cell membrane plays a vital role for the survival and 511 growth of the bacteria [45]. Reduction of membrane potential also increased the ROS 512 generation in treated cells and that is similar to previous reports [46]. ROS may oxidize 513 different cellular constituent including proteins, nucleic acid and lipid of the cell membrane.

514 Moreover, lipid oxidation may increase permeability of membrane as we found significant 515 uptake of PI in treated cells than untreated cells. Ultimately damage to membrane may leads 
516 to release of cellular constituents and death of the bacterial cells. This clinical MRSA was

517 found to be intermediate resistance to vancomycin. We found combination treatment of $1 / 4$

518 MIC of both vancomycin and ZnNPs-PK completely inhibited the growth of bacteria. This

519 may indicate synergistic activity of ZnNPs-PK and vancomycin in combination against

520 MRSA. Finally, it is important to understand biosafe nature of the prepared nanoparticles. the

521 IC90 concentration of nanoparticles were five times higher than the MIC of MRSA. The ratio

522 of IC90 $\mathrm{HaCaT}_{\text {and MIC }}$ MRSA showed that ZnNPs-PK is selectively cytotoxic towards MRSA as

523 the concentration required to inhibit bacterial growth is much lesser, i.e., 5-fold less than

524 IC90 HaCaT.

525 Conclusions:

526 This study concluded that ZnNPs-PK possesses bactericidal, anti-biofilms, anti-motility and 527 anti-virulence properties against MRSA. ZnNPs-PK targets cell membrane and increased 528 oxidative stress as its mode of action against MRSA. It increased the vancomycin sensitivity 529 of our clinical MRSA strain. ZnNPs-PK was found to nontoxic with selective bactericidal 530 properties. In a nutshell, ZnNPs-PK can be used for comprehensive treatment of diseases 531 associated with MRSA.

\section{3. Acknowledgements:}

533 The authors are thankful to Chancellor, Techno India University, West Bengal for providing

534 the necessary infrastructural and laboratory facilities. The authors are also thankful to Dr.

535 Ritesh Tiwari for supporting flow cytometry facilities at Centre for research in Nanoscience

536 and Nanotechnology (CRNN), University of Calcutta.

\section{Conflict of interest:}

538 The authors declare that there is no conflict of interests regarding the publication of this 539 paper. 
541

[1] J. P. O'Gara, 'Into the storm: Chasing the opportunistic pathogen Staphylococcus aureus from skin colonisation to life-threatening infections', Environ. Microbiol., vol. 19, no. 10, pp. 3823-3833, 2017.

[2] M. E. A. de Kraker, P. G. Davey, H. Grundmann, and BURDEN study group, 'Mortality and hospital stay associated with resistant Staphylococcus aureus and Escherichia coli bacteremia: estimating the burden of antibiotic resistance in Europe', PLoS Med., vol. 8, no. 10, p. e1001104, Oct. 2011.

[3] H. C. Neu, 'The crisis in antibiotic resistance', Science, vol. 257, no. 5073, pp. 10641073, Aug. 1992.

[4] M. Z. David and R. S. Daum, 'Update on Epidemiology and Treatment of MRSA Infections in Children', Curr Pediatr Rep, vol. 1, no. 3, pp. 170-181, Sep. 2013.

[5] S. Monecke et al., "A field guide to pandemic, epidemic and sporadic clones of methicillin-resistant Staphylococcus aureus', PLoS ONE, vol. 6, no. 4, p. e17936, Apr. 2011.

[6] R. J. Gordon and F. D. Lowy, 'Pathogenesis of Methicillin-Resistant Staphylococcus aureus Infection', CLIN INFECT DIS, vol. 46, no. S5, pp. S350-S359, Jun. 2008.

[7] M. K. Luther et al., 'Clinical and Genetic Risk Factors for Biofilm-Forming Staphylococcus aureus', Antimicrob Agents Chemother, vol. 62, no. 5, pp. e02252-17, /aac/62/5/e02252-17.atom, Mar. 2018.

[8] A. Kumar, A. Alam, M. Rani, N. Z. Ehtesham, and S. E. Hasnain, 'Biofilms: Survival and defense strategy for pathogens', Int. J. Med. Microbiol., vol. 307, no. 8, pp. 481489, Dec. 2017.

[9] U. Kadiyala, N. A. Kotov, and J. S. VanEpps, 'Antibacterial Metal Oxide Nanoparticles: Challenges in Interpreting the Literature', Curr. Pharm. Des., vol. 24, no. 8, pp. 896903, 2018.

[10] P. K. Mishra, H. Mishra, A. Ekielski, S. Talegaonkar, and B. Vaidya, 'Zinc oxide nanoparticles: a promising nanomaterial for biomedical applications', Drug Discov. Today, vol. 22, no. 12, pp. 1825-1834, 2017.

[11] A. Sirelkhatim et al., 'Review on Zinc Oxide Nanoparticles: Antibacterial Activity and Toxicity Mechanism', Nanomicro Lett, vol. 7, no. 3, pp. 219-242, 2015.

[12] R. Scheer and A. A. Fawzy, 'Antimicrobial treatment of pancreatin', Pharm Acta Helv, vol. 61, no. 9, pp. 253-256, 1986.

[13] B. Craigen, A. Dashiff, and D. E. Kadouri, 'The Use of Commercially Available AlphaAmylase Compounds to Inhibit and Remove Staphylococcus aureus Biofilms', Open Microbiol J, vol. 5, pp. 21-31, 2011.

[14] S. Shukla and Ts. Rao, 'Staphylococcus aureus biofilm removal by targeting biofilmassociated extracellular proteins', Indian J Med Res, vol. 146, no. 7, p. 1, 2017.

[15] V. Prabhawathi, T. Boobalan, P. M. Sivakumar, and M. Doble, 'Antibiofilm Properties of Interfacially Active Lipase Immobilized Porous Polycaprolactam Prepared by LB Technique', PLoS ONE, vol. 9, no. 5, p. e96152, May 2014.

[16] J. Schulz, A. Boklund, N. Toft, and T. Halasa, 'Risk-based eradication as a control measure to limit the spread of LA-MRSA among Danish pig herds - a simulation study', Sci Rep, vol. 9, no. 1, p. 13192, Sep. 2019.

[17] R. Herrera-Rivera, M. de la L. Olvera, and A. Maldonado, 'Synthesis of $\mathrm{ZnO}$ Nanopowders by the Homogeneous Precipitation Method: Use of Taguchi's Method for Analyzing the Effect of Different Variables', Journal of Nanomaterials, vol. 2017, pp. $1-9,2017$. 
604

605

606

607

608

609

610

611

612

613

614

615

616

617

618

619

620

621

622

623

624

625

626

627

628

629

630

631

632

633

634

635

[18] R. Mukherjee, M. Patra, D. Dutta, M. Banik, and T. Basu, 'Tetracycline-loaded calcium phosphate nanoparticle (Tet-CPNP): Rejuvenation of an obsolete antibiotic to further action', Biochim. Biophys. Acta, vol. 1860, no. 9, pp. 1929-1941, 2016.

[19] L. de Sousa Eduardo, T. C. Farias, S. B. Ferreira, P. B. Ferreira, Z. N. Lima, and S. B. Ferreira, 'Antibacterial Activity and Time-kill Kinetics of Positive Enantiomer of $\alpha$ pinene Against Strains of Staphylococcus aureus and Escherichia coli', Curr Top Med Chem, vol. 18, no. 11, pp. 917-924, 2018.

[20] D. Rubini et al., 'Essential oils from unexplored aromatic plants quench biofilm formation and virulence of Methicillin resistant Staphylococcus aureus', Microbial Pathogenesis, vol. 122, pp. 162-173, Sep. 2018.

[21] P. Chemmugil, P. T. V. Lakshmi, and A. Annamalai, 'Exploring Morin as an antiquorum sensing agent (anti-QSA) against resistant strains of Staphylococcus aureus', Microb. Pathog., vol. 127, pp. 304-315, Feb. 2019.

[22] J.-H. Lee, Y.-G. Kim, S. Yong Ryu, and J. Lee, 'Calcium-chelating alizarin and other anthraquinones inhibit biofilm formation and the hemolytic activity of Staphylococcus aureus', Sci Rep, vol. 6, no. 1, p. 19267, May 2016.

[23] C. Nithya, M. G. Devi, and S. Karutha Pandian, 'A novel compound from the marine bacterium Bacillus pumilus S6-15 inhibits biofilm formation in gram-positive and gramnegative species', Biofouling, vol. 27, no. 5, pp. 519-528, May 2011.

[24] D. Rubini et al., 'Chitosan extracted from marine biowaste mitigates staphyloxanthin production and biofilms of Methicillin- resistant Staphylococcus aureus', Food and Chemical Toxicology, vol. 118, pp. 733-744, Aug. 2018.

[25] K. Pakshir, M. Ravandeh, H. Khodadadi, M. Motamedifar, K. Zomorodian, and S. Alipour, 'Evaluation of Exoenzyme Activities, Biofilm Formation, and Co-hemolytic Effect in Clinical Isolates of Candida parapsilosis Species Complex', J Glob Infect Dis, vol. 10, no. 3, pp. 163-165, Sep. 2018.

[26] C. Kong, C.-F. Chee, K. Richter, N. Thomas, N. Abd. Rahman, and S. Nathan, 'Suppression of Staphylococcus aureus biofilm formation and virulence by a benzimidazole derivative, UM-C162', Sci Rep, vol. 8, no. 1, p. 2758, Dec. 2018.

[27] S. Kumari, S. Rajit Prasad, D. Mandal, and P. Das, 'Carbon dot-DNA-protoporphyrin hybrid hydrogel for sustained photoinduced antimicrobial activity', J Colloid Interface Sci, vol. 553, pp. 228-238, Oct. 2019.

[28] K. Sood, J. Kaur, H. Singh, S. Kumar Arya, and M. Khatri, 'Comparative toxicity evaluation of graphene oxide (GO) and zinc oxide $(\mathrm{ZnO})$ nanoparticles on Drosophila melanogaster', Toxicol Rep, vol. 6, pp. 768-781, 2019.

[29] Q. Yi et al., 'Singlet Oxygen Triggered by Superoxide Radicals in a Molybdenum Cocatalytic Fenton Reaction with Enhanced REDOX Activity in the Environment', Environ. Sci. Technol., vol. 53, no. 16, pp. 9725-9733, Aug. 2019.

[30] A. Ganguli, D. Choudhury, S. Datta, S. Bhattacharya, and G. Chakrabarti, 'Inhibition of autophagy by chloroquine potentiates synergistically anti-cancer property of artemisinin by promoting ROS dependent apoptosis', Biochimie, vol. 107 Pt B, pp. 338-349, Dec. 2014.

[31] N. R. Cutler, J. J. Sramek, and P. K. Narang, Eds., Pharmacodynamics and drug development: perspectives in clinical pharmacology. Chichester: Wiley, 1994.

[32] C. Kaito and K. Sekimizu, 'Colony Spreading in Staphylococcus aureus', Journal of Bacteriology, vol. 189, no. 6, pp. 2553-2557, Mar. 2007.

[33] O. Ates, 'Systems Biology of Microbial Exopolysaccharides Production', Front Bioeng Biotechnol, vol. 3, p. 200, 2015. 
642

643

644

645

646

647

648

649

650

651

652

653

654

655

656

657

658

659

660

661

662

663

664

665

666

667

668

669

670

671

672

673

674

675

676

677

678

[34] A. Clauditz, A. Resch, K.-P. Wieland, A. Peschel, and F. Götz, 'Staphyloxanthin plays a role in the fitness of Staphylococcus aureus and its ability to cope with oxidative stress', Infect. Immun., vol. 74, no. 8, pp. 4950-4953, Aug. 2006.

[35] B. Fournier and D. J. Philpott, 'Recognition of Staphylococcus aureus by the Innate Immune System', Clinical Microbiology Reviews, vol. 18, no. 3, pp. 521-540, Jul. 2005.

[36] H. Strahl and L. W. Hamoen, 'Membrane potential is important for bacterial cell division', Proceedings of the National Academy of Sciences, vol. 107, no. 27, pp. 12281-12286, Jul. 2010.

[37] P. Sahariah et al., 'Antimicrobial peptide shows enhanced activity and reduced toxicity upon grafting to chitosan polymers', Chem. Commun. (Camb.), vol. 51, no. 58, pp. 11611-11614, Jul. 2015.

[38] S. Y. C. Tong, J. S. Davis, E. Eichenberger, T. L. Holland, and V. G. Fowler, 'Staphylococcus aureus Infections: Epidemiology, Pathophysiology, Clinical Manifestations, and Management', Clin. Microbiol. Rev., vol. 28, no. 3, pp. 603-661, Jul. 2015.

[39] S. Vora, 'Acute renal failure due to vancomycin toxicity in the setting of unmonitored vancomycin infusion', Proc (Bayl Univ Med Cent), vol. 29, no. 4, pp. 412-413, Oct. 2016.

[40] M. Piechota, B. Kot, A. Frankowska-Maciejewska, A. Grużewska, and A. WoźniakKosek, 'Biofilm Formation by Methicillin-Resistant and Methicillin-Sensitive Staphylococcus aureus Strains from Hospitalized Patients in Poland', BioMed Research International, vol. 2018, pp. 1-7, Dec. 2018.

[41] B. F. Ricciardi, G. Muthukrishnan, E. Masters, M. Ninomiya, C. C. Lee, and E. M. Schwarz, 'Staphylococcus aureus Evasion of Host Immunity in the Setting of Prosthetic Joint Infection: Biofilm and Beyond', Curr Rev Musculoskelet Med, vol. 11, no. 3, pp. 389-400, Sep. 2018.

[42] B. Parrino, P. Diana, G. Cirrincione, and S. Cascioferro, 'Bacterial Biofilm Inhibition in the Development of Effective Anti-Virulence Strategy', Open Med Chem J, vol. 12, pp. 84-87, 2018.

[43] E. Hodille, W. Rose, B. A. Diep, S. Goutelle, G. Lina, and O. Dumitrescu, 'The Role of Antibiotics in Modulating Virulence in Staphylococcus aureus', Clin. Microbiol. Rev., vol. 30, no. 4, pp. 887-917, Oct. 2017.

[44] K. S. Siddiqi, A. Ur Rahman, null Tajuddin, and A. Husen, 'Properties of Zinc Oxide Nanoparticles and Their Activity Against Microbes', Nanoscale Res Lett, vol. 13, no. 1, p. 141, May 2018.

[45] O. M. Bondarenko, M. Sihtmäe, J. Kuzmičiova, L. Ragelienè, A. Kahru, and R. Daugelavičius, 'Plasma membrane is the target of rapid antibacterial action of silver nanoparticles in Escherichia coli and Pseudomonas aeruginosa', Int J Nanomedicine, vol. 13, pp. 6779-6790, 2018.

[46] T. Wang, I. El Meouche, and M. J. Dunlop, 'Bacterial persistence induced by salicylate via reactive oxygen species', Sci Rep, vol. 7, p. 43839, 102017.

\section{Figure Legends:}


679 Fig.1 - Characterizations of ZnNPs and ZnNPs-PK. (A) Comparative UV-VIS spectra of 680 pancreatin, ZnNPs and ZnNPs-PK by UV-spectrophotometer. (B) Comparative analysis of 681 ZnNPs (B1) and ZnNPs-PK(B2) by Dynamic Light Scattering (DLS).

682 Fig.2- Antimicrobial activities of ZnNPs-PK on MRSA. (A)Graphical representation of 683 comparative antimicrobial activity of ZnNPs and ZnNPs-PK (B) Death Kinetics of MRSA in 684 presence and absence of ZnNPs-PK.

685 Fig.3 - Anti-biofilms activity by ZnNPs-PK against MRSA.(A)Tabular representation of 686 effect of ZnNPs-PK on the viability of sessile cells and biofilm biomass of initial and mature 687 biofilms (B) Light (B1 and B2) and confocal microscopic (B3 and B4) observation of ZnNPs$688 \quad$ PK induced biofilm inhibition

689 Fig.4 - Anti-motility activity by ZnNPs-PK against MRSA (A) Pictorial representation of 690 inhibition of sliding motility (A1 and A2) and colony motility (A3 and A4) of MRSA by 691 ZnNPs-PK.

692 Fig.5 - Anti-virulence properties of ZnNPs-PK against MRSA (A)Pictorial representation of 693 inhibition of slime production of MRSA by ZnNPs-PK. (A1) represents untreated cells. (A2) 694 represents treated cells. (B) Pictorial representation of plasma clamping ability (A3 and A4) 695 of MRSA by ZnNPs-PK. (B1) blood plasma with untreated MRSA cells. (B2) blood plasma 696 with treated MRSA cells.

697 Fig.6 - (A) Tabular representation of effect of ZnNPs-PK on exopolysaccharide production, 698 staphyloxanthin production and hemolytic activity of MRSA. (B) ROS generation was flow 699 cytometrically evaluated in untreated and ZnNPs-PK treated MRSA cells by using DCFDA.

$700 \quad$ Fig.7 - Mechanism of action of ZnNPs-PK against MRSA (A) Membrane potential was flow 701 cytometrically evaluated in untreated and ZnNPs-PK treated MRSA cells by using Rh123 (B) 
702 Cell membrane damage was flow cytometrically evaluated in untreated and ZnNPs-PK 703 treated MRSA cells by PI uptake

704 Fig.8 - Ex vivo study and combination study of ZnNPs-PK (A) Graphical representation of 705 bioburden of MRSA cells untreated and treated with ZnNPs-PK in porcine skin model. 706 (B)Graphical representation of combination activity of ZnNPs-PK and Vancomycin against 707 MRSA

708

709

710

711

712

713

714

715

716

717

718

719

720

721

722

723

724 
Figures:

A

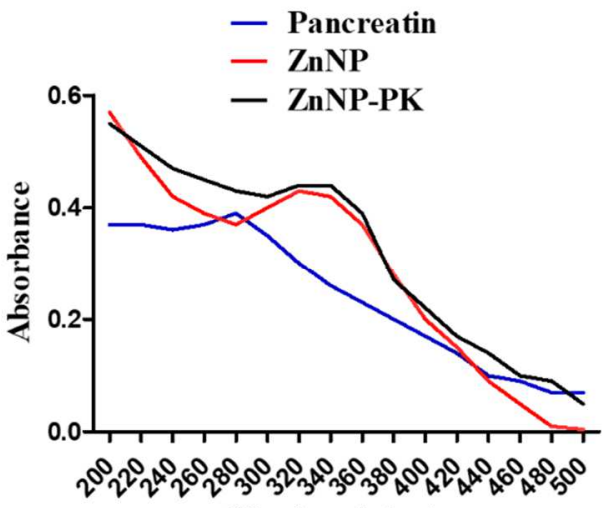

726

727

A

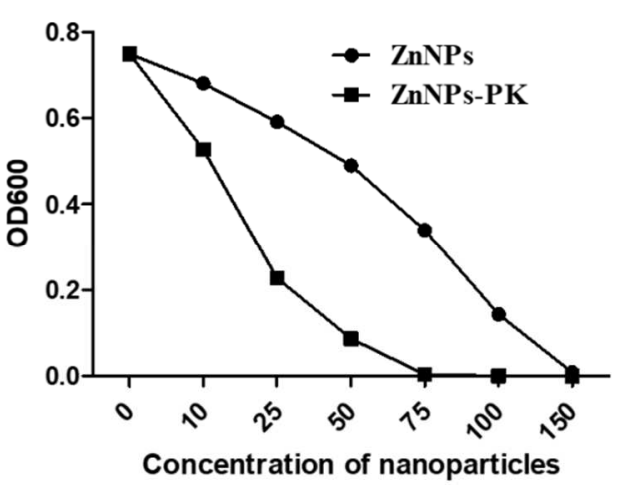

B

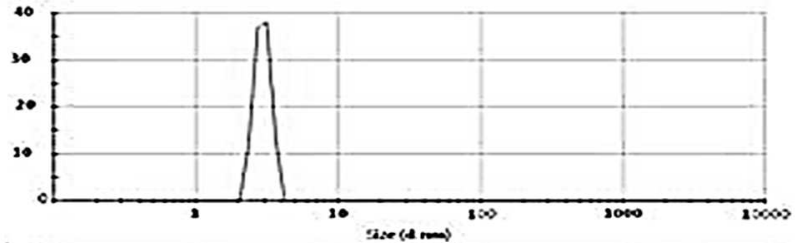

C

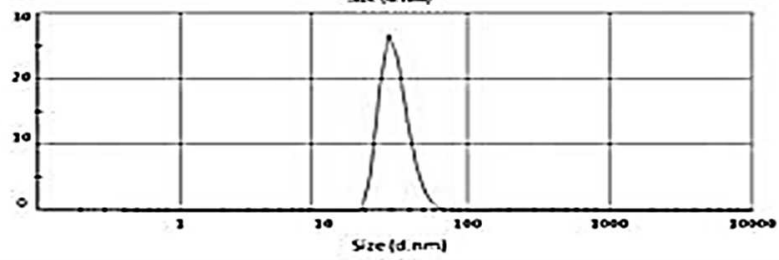

Fig.1

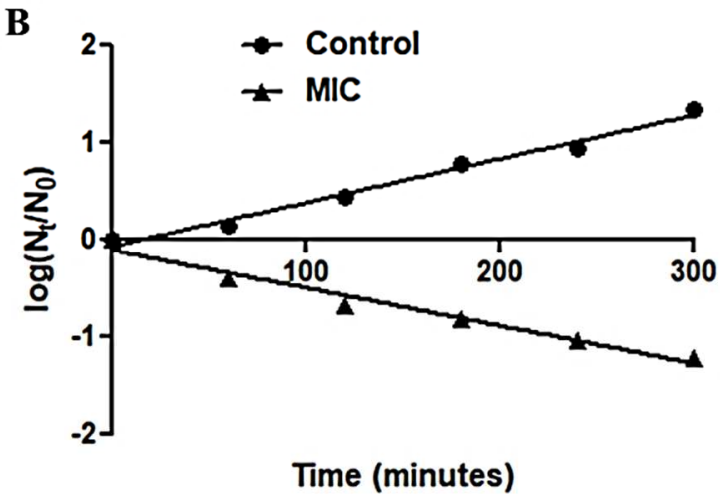

Fig.2 


\begin{tabular}{|l|c|c|c|c|}
\cline { 2 - 5 } \multicolumn{1}{c|}{} & \multicolumn{2}{c|}{ Initial Biofilm } & \multicolumn{2}{c|}{ Mature Biofilm } \\
\cline { 2 - 5 } \multicolumn{1}{c|}{} & CFU/ml & Biomass & CFU/ml & Biomass \\
\hline Control & $8.38 \pm 0.67$ & 100 & $8.61 \pm 0.23$ & 100 \\
\hline $1 / 2$ MIC & $6.6 \pm 0.21$ & $38 \pm 3.2$ & $6.8 \pm 0.07$ & $51 \pm 1.1$ \\
\hline MIC & $4.2 \pm 0.31$ & $17 \pm 2.7$ & $5.1 \pm 0.15$ & $32 \pm 2.4$ \\
\hline
\end{tabular}
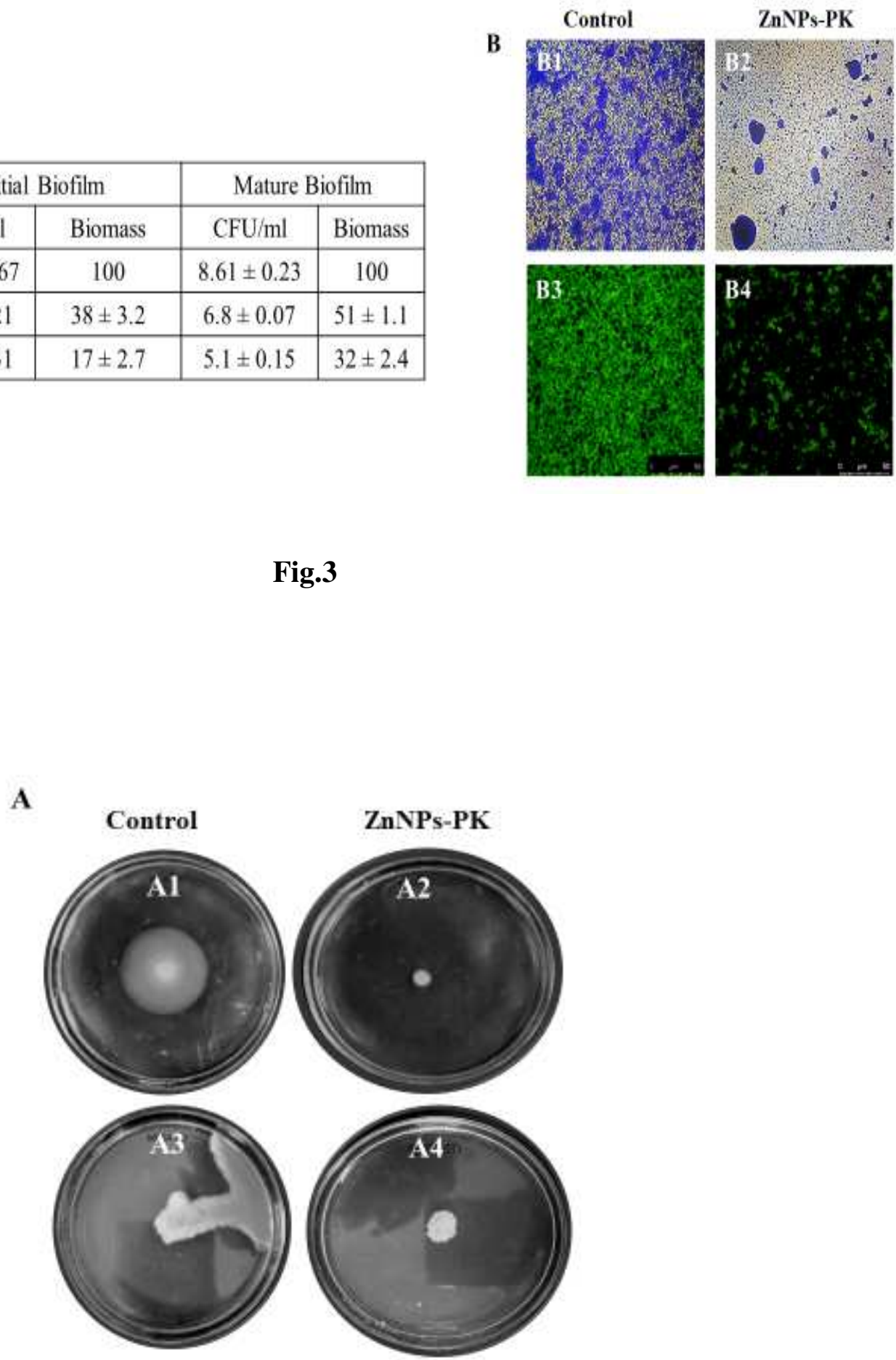

735

Fig. 4 
A

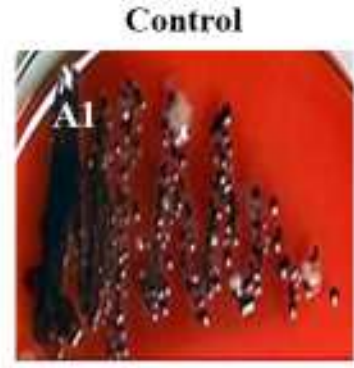

ZnNPs-PK

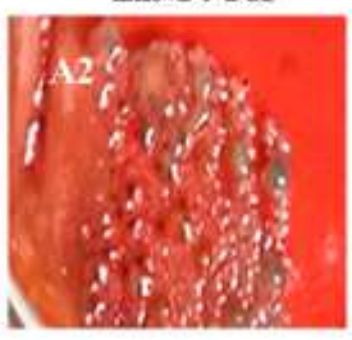

B

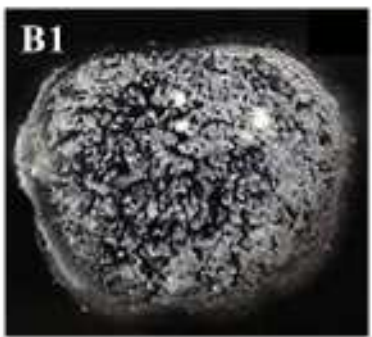

\section{B2}

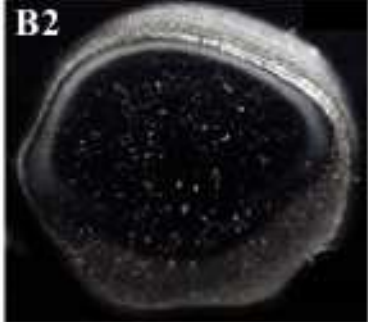

Fig. 5

A

\begin{tabular}{|c|c|c|}
\cline { 2 - 3 } \multicolumn{1}{c|}{} & Control & MIC \\
\hline $\begin{array}{c}\text { Exopolysaccharide } \\
\text { (OD495nm/ 10 } \\
\text { Cells) }\end{array}$ & $0.168 \pm 0.015$ & $0.062 \pm 0.009$ \\
\hline $\begin{array}{c}\text { Staphyloxanthine } \\
\text { (OD462nm/10 } \\
\text { Cells) }\end{array}$ & $0.992 \pm 0.035$ & $0.367 \pm 0.053$ \\
\hline $\begin{array}{c}\text { Hemolytic Potential } \\
\text { (\%) }\end{array}$ & 100 & $17 \pm 2.1$ \\
\hline
\end{tabular}

741

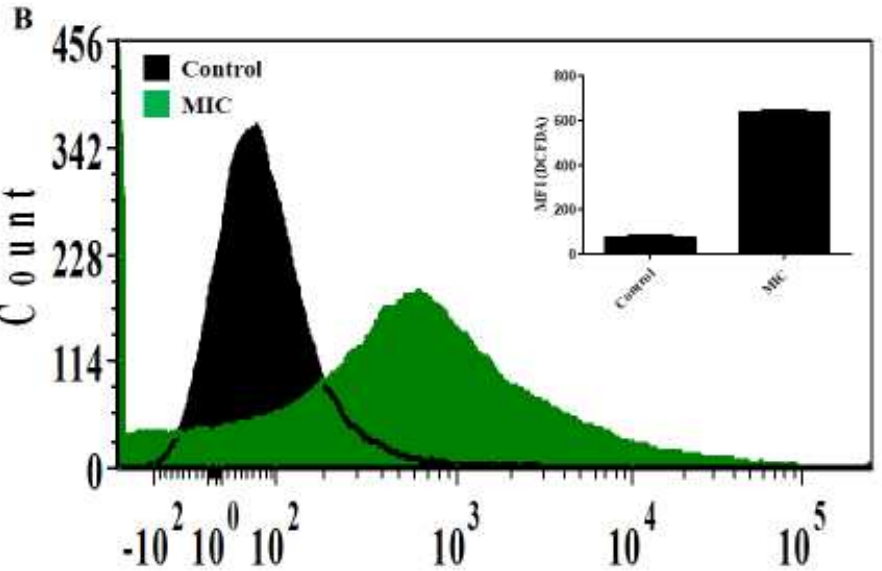

742

Fig.6

743

744 
A

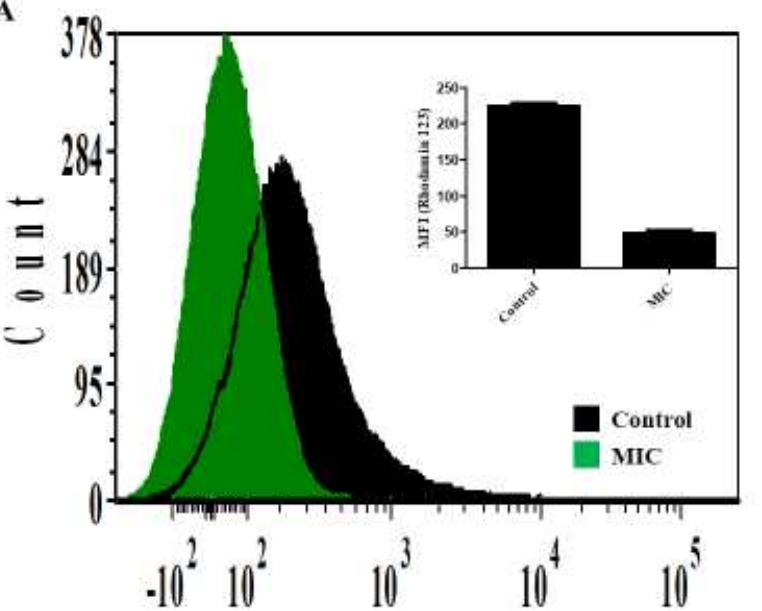

746

A

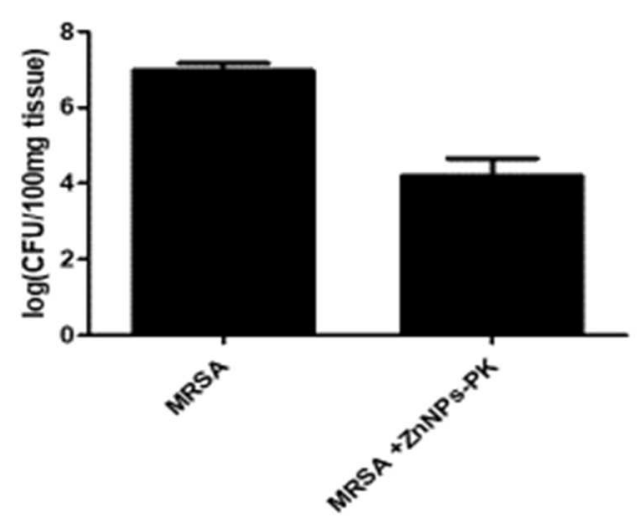

Fig.7
B
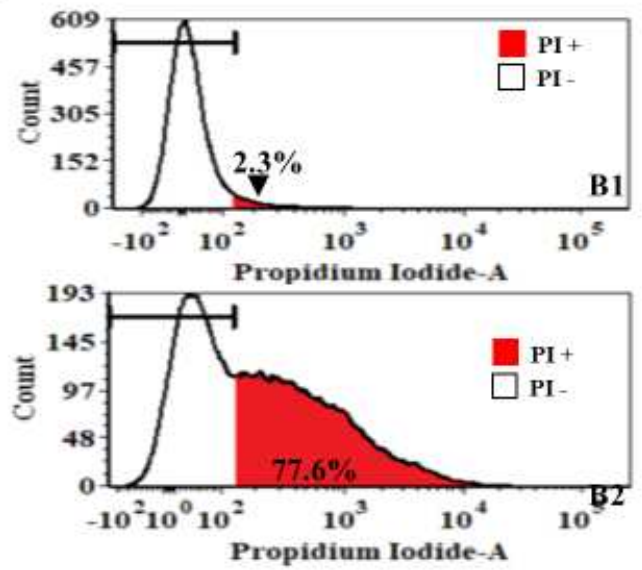

B

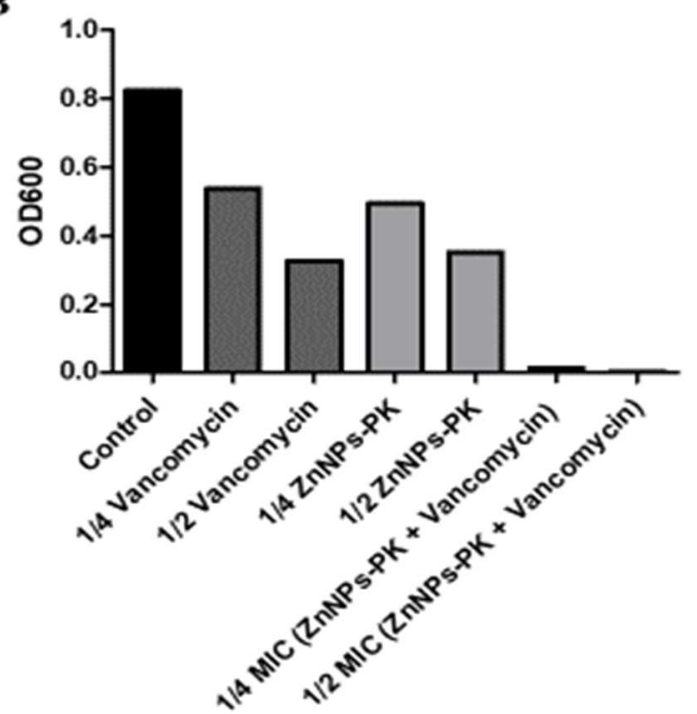

Fig. 8 


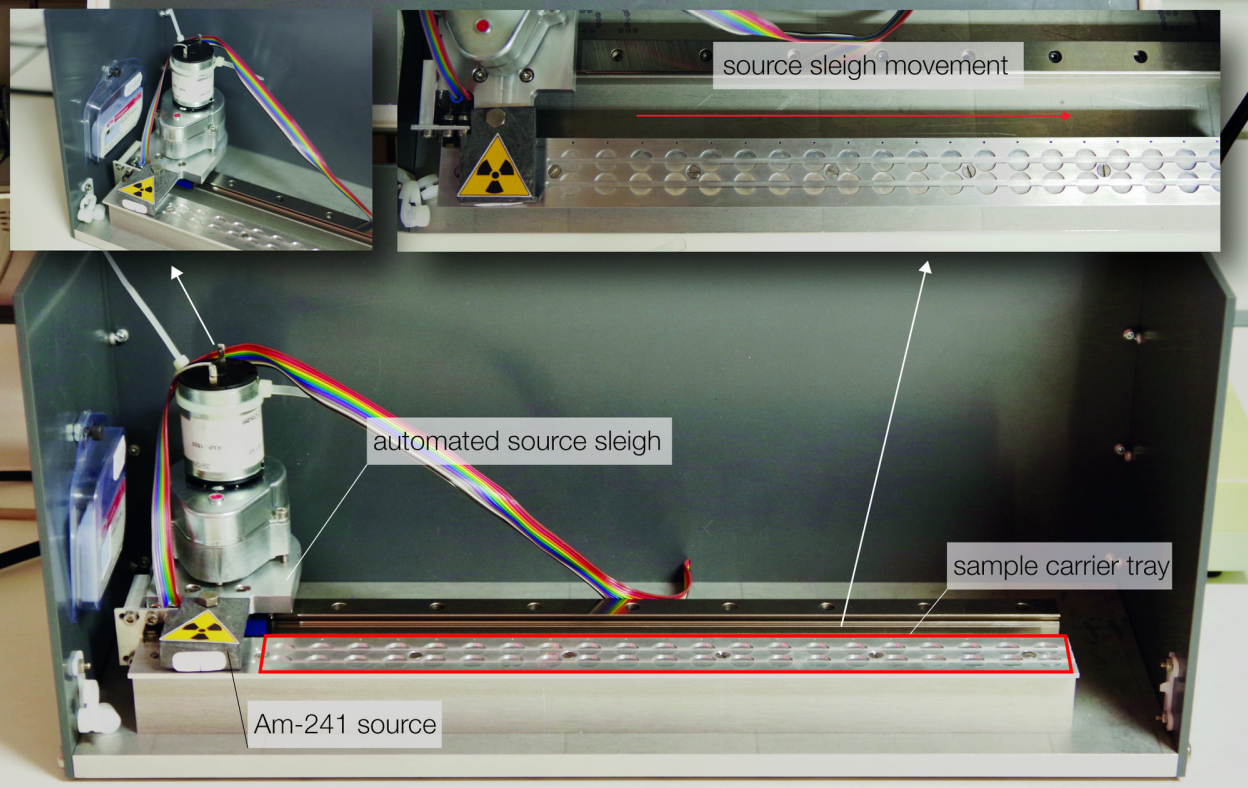

Dimension: $115 \times 270 \times 124 \mathrm{~mm}$ (length $\mathrm{x}$ width $\mathrm{x}$ height) 

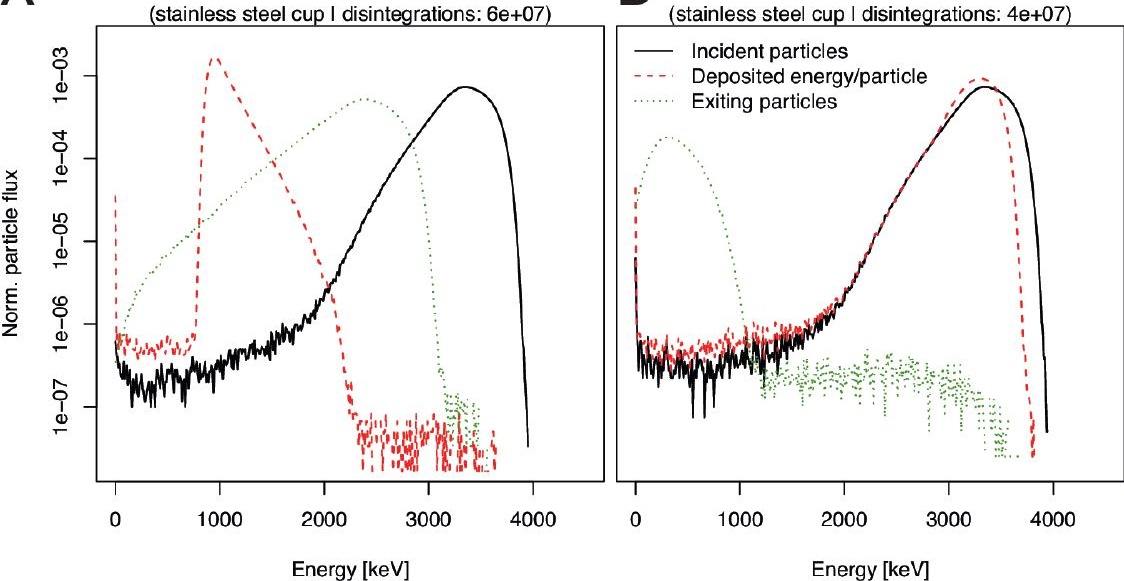

Energy [keV]

Energy [keV] 

A BDX16651 I pos. 11 I alpha-irrd. 0

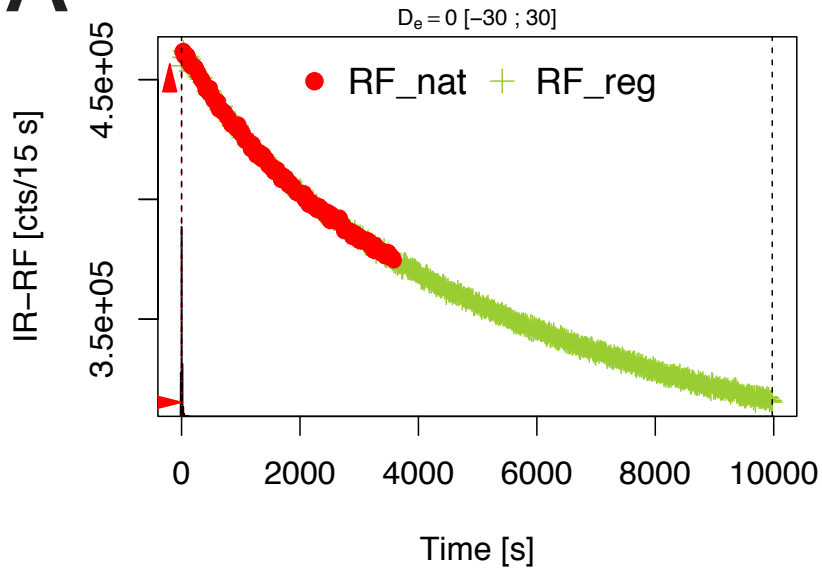

BDX16646 I pos. 4 I alpha-irrd. 25200

10
0
+
0
0
0

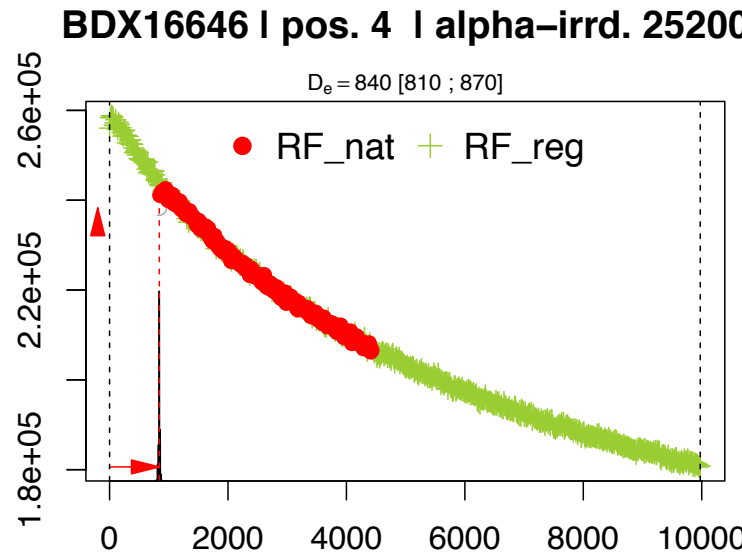

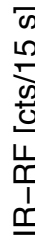

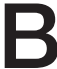

Time [s] 


\section{Alpha-flux Response Curve}

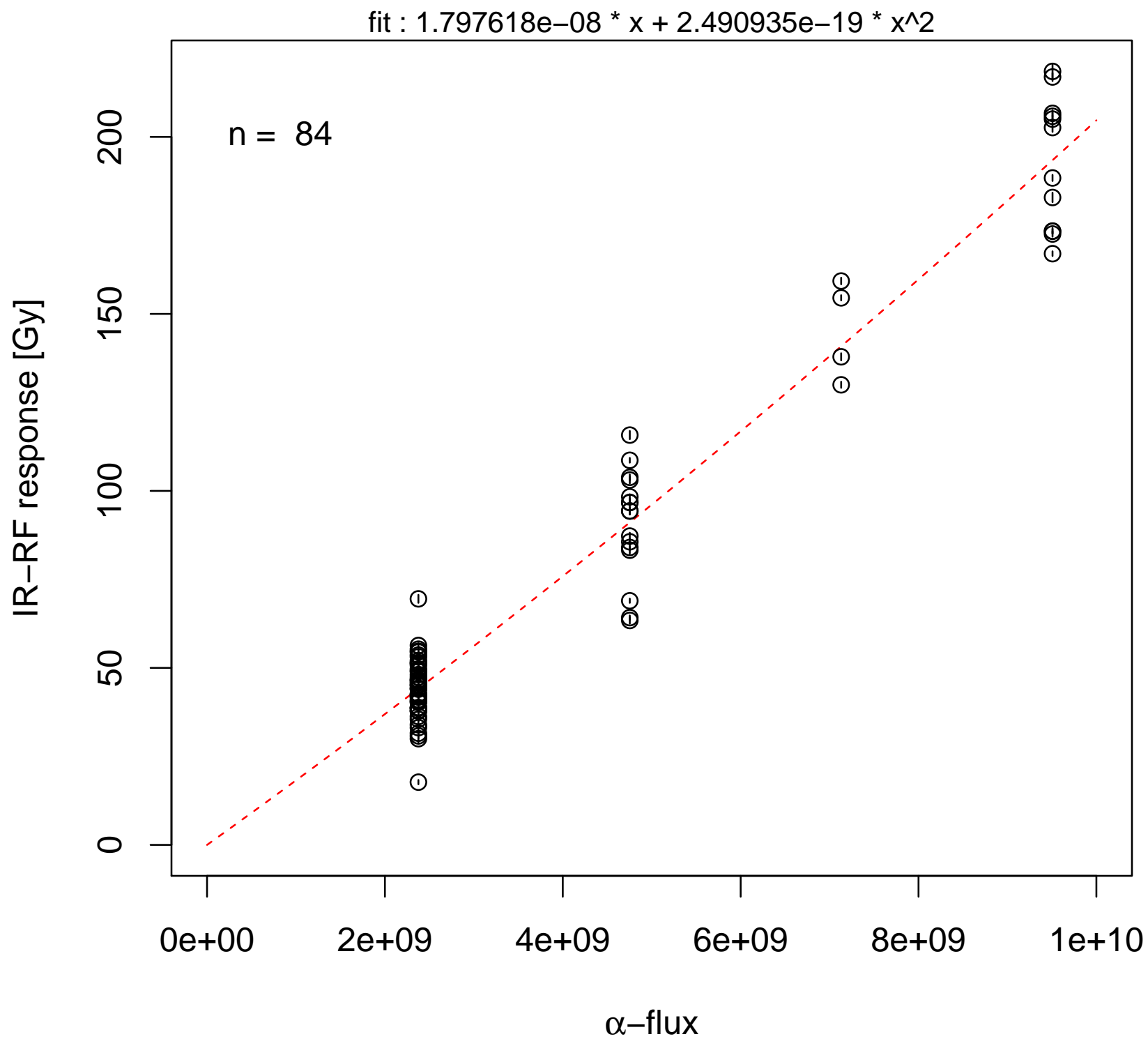




\section{$S_{\alpha}$-values over Time}

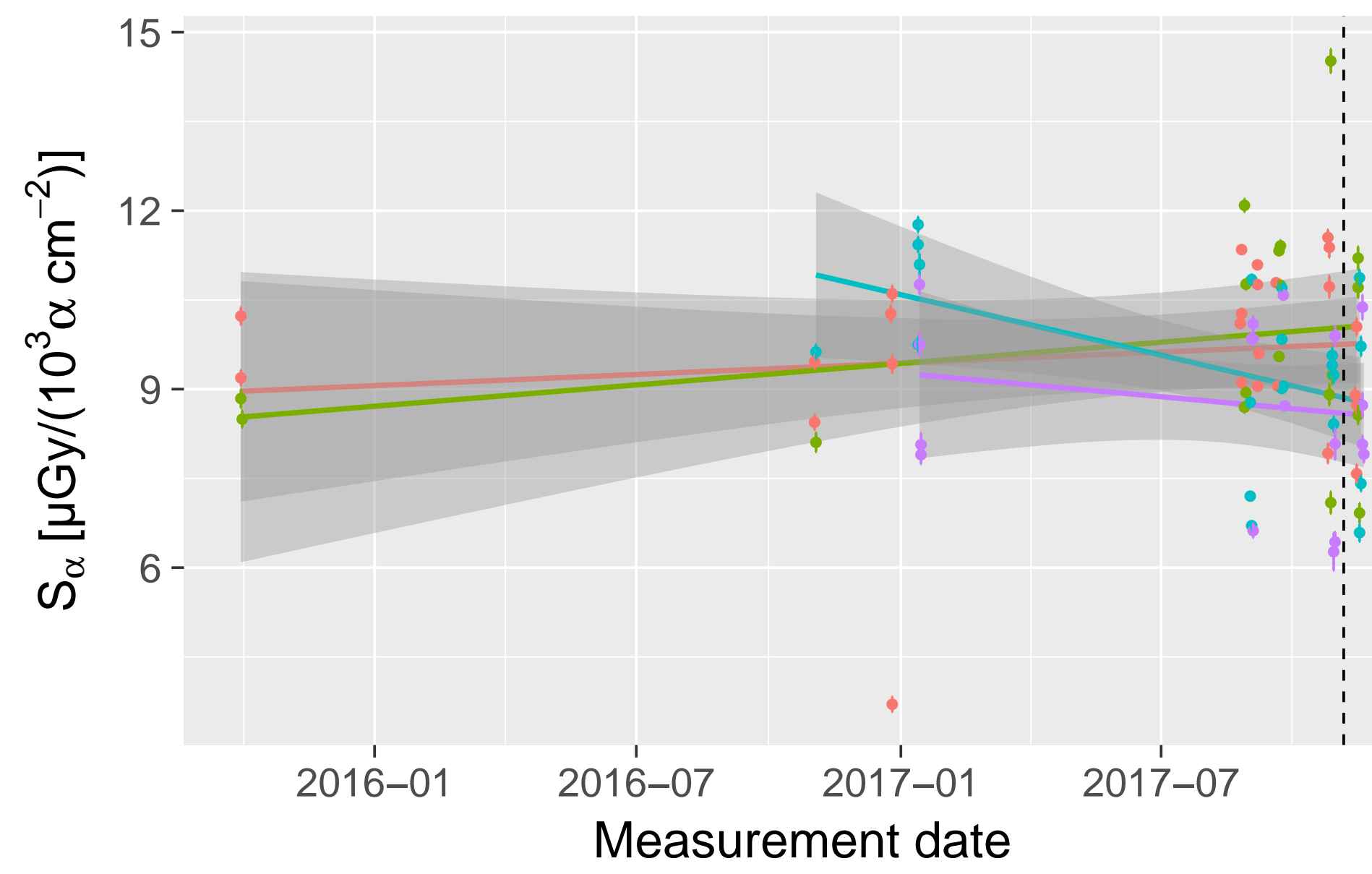

Sample $-\mathrm{BDX} 16646-\mathrm{BDX} 16650-\mathrm{BDX} 16651-\mathrm{BT706}$ 
$\mathrm{n}=84 \mid$ mean $=9.26 \mid$ abs. $\mathrm{sd}=1.62 \mid$ rel. $\mathrm{sd}=17.23 \%$
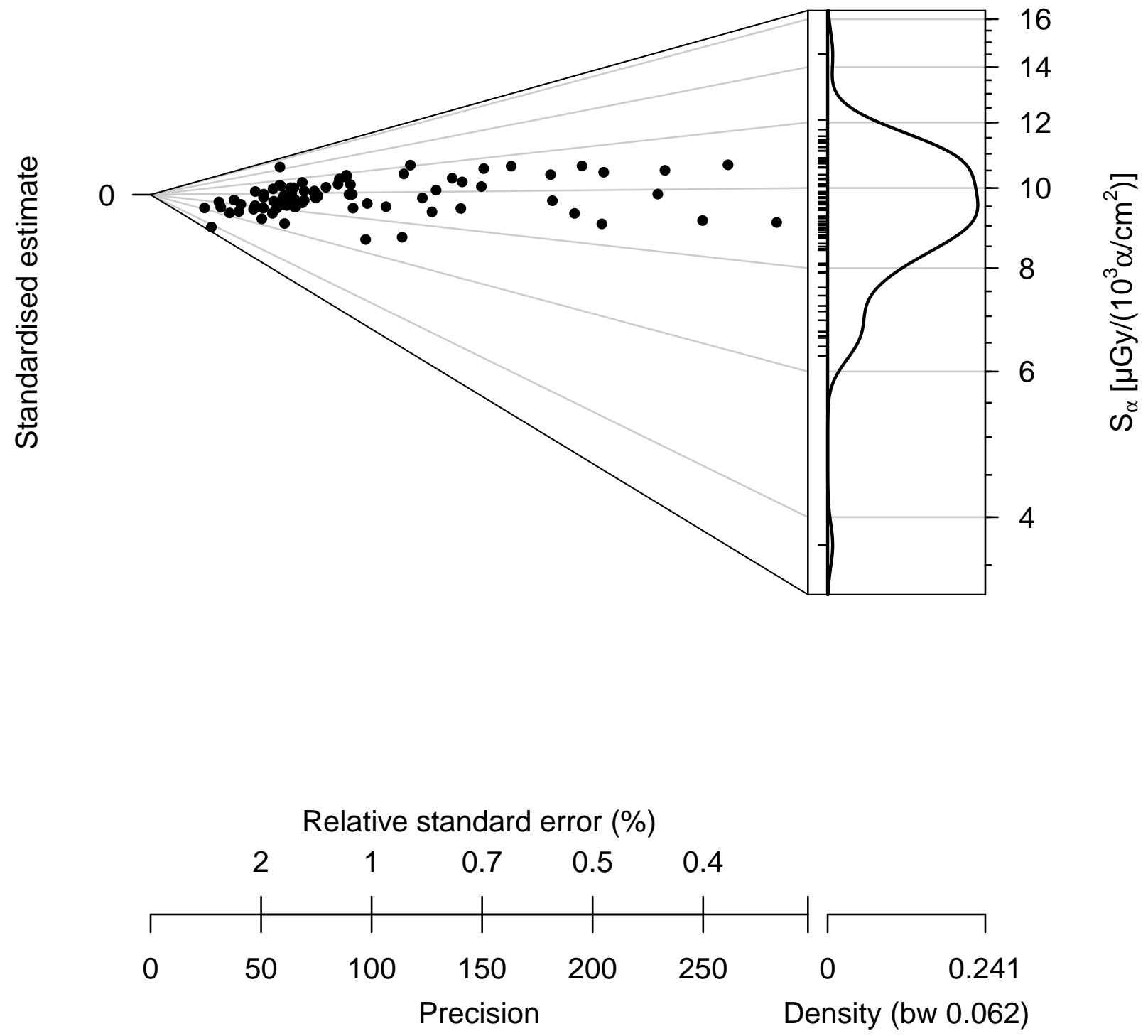
Table 1

\begin{tabular}{lcccc}
\hline SAMPLE & $\mathbf{N}$ & $\begin{array}{c}\boldsymbol{S}_{\alpha} \\
{\left[\mu \mathrm{Gy} /\left(10^{3} \alpha \mathrm{cm}^{-2}\right)\right]}\end{array}$ & $\begin{array}{c}\mathbf{S E}\left(\boldsymbol{S}_{\alpha}\right) \\
{\left[\mu \mathrm{Gy} /\left(10^{3} \alpha \mathrm{cm}^{-2}\right)\right]}\end{array}$ & $\begin{array}{c}\text { CV } \\
{[\%]}\end{array}$ \\
\hline BDX16646 & $26 / 28$ & 10.0 & 1.2 & 12.4 \\
BDX16650 & $19 / 19$ & 10.0 & 1.3 & 13.0 \\
BDX16651 & $21 / 23$ & 9.2 & 1.2 & 12.8 \\
BT706 & $18 / 22$ & 9.0 & 1.2 & 13.2 \\
\hline
\end{tabular}

Note: All values are given as error weighted mean. SE is the standard deviation of the sampling distribution. 